\title{
Increased breath ethane and pentane concentrations in currently unmedicated patients with schizophrenia
}

\author{
Brian M. Ross ${ }^{1}$, Sandeep Shah ${ }^{2}$, Malcolm Peet ${ }^{3}$ \\ ${ }^{1}$ Northern Ontario School of Medicine, Thunder Bay, Ontario, Canada; \\ ${ }^{2}$ Sumandeep Vidyapeeth, AT\&PO: Piparia, Vadodara, India; \\ ${ }^{3}$ University of Sheffield, Sheffield, United Kingdom. \\ Email: brian.ross@nosm.ca
}

Received 19 March 2011; revised 21 April 2011; accepted 28 April 2011.

\begin{abstract}
Schizophrenia is a common and debilitating mental illness. The disorder is thought to be developmental in origin, with oxidative stress being implicated as possible pathophysiological mechanism. Breath alkanes provide a non-invasive means to assess oxidative stress, with ethane levels reportedly increased in medicated patients with schizophrenia. It is possible, however, that the psychotropic medications used to treat the disorder result in elevated breath ethane levels. We have therefore measured the concentration of ethane and pentane, markers of oxidative stress, in the breath of currently unmedicated patients with schizophrenia. Alveolar breath samples were collected, applied to thermal desorption tubes, and analyzed using a combination of two-stage thermal desorption, gas chromatography and mass spectrometry. Compared to healthy controls ethane and pentane levels were found to be elevated in patients with schizophrenia, while levels of butane were normal. Our data support the notion that oxidative stress is increased in schizophrenia and that this is unlikely to be a consequence of antipsychotic medications. In addition, breath alkane analysis may represent a rapid and non-invasive means to monitor oxidative stress occurring in schizophrenia.
\end{abstract}

Keywords: Breath Analysis; Alkane, Schizophrenia; Ethane; Pentane

\section{INTRODUCTION}

Schizophrenia is a serious mental illness which first presents in late adolescence or early adulthood. [1] The disorder, which affects approximately $1 \%$ of the population, is characterized by a range of symptoms including delusions, hallucinations, disordered thoughts and avolition. [1,2] Current treatments have limited efficacy in many patients, with the development of more effective therapies being hampered by a lack of understanding of the pathophysiological mechanism (s) at work. [1] An emerging hypothesis states that disordered oxidative metabolism leads to the brain dysfunction which underlies the disorder. [3] This process, termed oxidative stress, comprises the reactions between metabolically generated free radicals (highly reactive molecules possessing unpaired electrons) and cellular constituents such as DNA, lipids and proteins. [4] The reactions are generally deleterious, leading to a loss of, or altered, function of the cell which, if severe enough, can result in cell death. [4] To combat this process cells possess various antioxidant defenses the purpose of which is to detoxify free radicals. These defenses include enzymes, such as superoxide dismutase, glutathione peroxidase and catalase, and antioxidant compounds, such as vitamin $\mathrm{E}$, which preferentially react with free radicals hence preserving other cellular constituents. [4] As such the degree of oxidative stress a cell experiences is determined by the balance between oxidative and anti-oxidative factors.

In schizophrenia, a number of lines of evidence have suggested that oxidative stress is abnormal $[3,5,6]$. Since direct measurement of the short lived and reactive free radicals is extremely difficult, most information regarding oxidative stress in schizophrenia derives from the assay of specific components of the anti-oxidant system and/or measurement of the chemical products of oxidative damage. While generally supportive, when examined in detail the findings are somewhat inconsistent. For example, peripheral superoxide dismutase activity, an enzyme which detoxifies superoxide radicals, is reported to be either decreased, increased or unchanged, with similar variability of results being reported for catalase and glutathione peroxidise. [3,5-13] It is notable, however, that when the system is considered as a whole, using methods which assess total antioxidant capacity, a consistent reduction in antioxidant activity in schizophrenia 
emerges, indicating an overall elevated susceptibility to free radical damage in the disorder. [14-16] This is supported by several reports of increased levels of DNA, protein and lipid oxidation products in patients with schizophrenia. [17-21] Moreover, the use of an animal model of oxidative stress has indicated that free radical over-activity effects brain development and function, supporting the biological plausibility of oxidative stress playing a role in the disease. [22,23]

As such, monitoring oxidative stress in patients may have some clinical utility. Relating the normally utilised peripheral measures of oxidative stress to that in the brain is challenging however, although evidence of oxidative damage has been detected post mortem in the cortex and hippocampus of patients with schizophrenia. $[17,18,21]$ A recent study has correlated an index of systemic oxidative stress with changes in brain metabolism investigated using in vivo ${ }^{31} \mathrm{P}$ magnetic resonance spectroscopy. [24] The marker utilised was ethane, a terminal product of the oxidation of omega-3 polyunsaturated fatty acids [4], which has been shown to be elevated in schizophrenia in two previous studies [25,26], a finding supportive of the occurrence of increased oxidative stress. The other class of polyunsaturated fatty acids, omega- 6 fatty acids, also give rise to an alkane oxidation product which differs from that of omega-3 fatty acids, this being pentane [4]. A generalised increase in oxidative stress in schizophrenia would predict that breath concentrations of pentane should be increased although the status of this compound in schizophrenia is currently unknown. Since ethane and pentane are both highly volatile they have the useful feature of equilibrating rapidly into the bloodstream, and subsequently crossing the alveolar membrane to be exhaled in the breath where they can be detected using a variety of analytical approaches including gas chromatography and various forms of chemical ionisation mass spectrometry. [27-29] So-called breath analysis is an emerging methodology which, being noninvasive and rapid, is ideally suited to clinical monitoring. [30] Given the correlation of breath ethane with brain metabolism [24], measuring the breath concentration of this compound may represent a useful means to examine oxidative stress in schizophrenia. Studies utilising breath ethane in schizophrenia should be considered preliminary, however, since only medicated patients with the disorder have been included. Indeed, recent reports have highlighted that pharmaceuticals can alter the chemical makeup of breath, and in fact can give rise to volatile metabolites raising the possibility that ethane may derive directly from such medications. [31,32] In addition, some antipsychotic medications, in particular clozapine and haloperidol, may actually cause increased oxidative stress [33-36], an effect which could again result in altered breath ethane abundance. As such, it presently cannot be ruled out that elevated breath ethane in schizophrenia is due to medication. In this paper we have therefore investigated breath concentrations of both ethane and pentane, as well as butane as a comparator, in a currently un-medicated population of patients with schizophrenia.

\section{METHODS}

\subsection{Participants}

Subjects were recruited by invitation having given written informed consent under a protocol approved by the institutional ethics committee. Patients with schizophrenia all had a clinical diagnosis of schizophrenia $(n=28)$ according to DSM-IV criteria. [37] Patients had not been in receipt of psychotropic medication for three weeks prior to participating in the study but had received medication prior to that (no patients were withdrawn from their medication for the purpose of this study; rather non-compliance with treatment had occurred). Healthy controls $(n=15)$ had neither history of mental illness nor any medicinal intake of any kind for the previous two weeks, and had no history of any psychotropic drug intake. Almost all subjects were smokers with only four non-smokers in the schizophrenia group and two in the healthy control group with the rate of smoking not differing between groups ( $\chi^{2}$ test; $P>0.05$ ). No subject had smoked within the 2 hours prior to breath collection. No subjects had any potentially confounding medical illness. The age of patients with schizophrenia $(33 \pm 6$ years $[$ mean $\pm \mathrm{sd}]$ ) did not differ significantly (two-tailed, unpaired t-test; $P>0.05)$ from that of the healthy control group ( $34 \pm 7$ years). In addition the proportion of males to females in each group (15 females/13 males in the schizophrenia group vs. 8 males/7 females in the healthy control group) did not differ significantly ( $\chi^{2}$ test; $P>$ $0.05)$.

\subsection{Breath Sampling}

Seated subjects were asked to breathe normally for 3 breaths and then exhale fully into a collection bag constructed in our laboratory. The collection bag was made from PTFE with dimensions of approximately $10 \mathrm{~cm} \times$ $15 \mathrm{~cm}$ and a volume of approximately $200 \mathrm{ml}$. The bag had both an inlet and outlet, with air from the inlet entering the bag via a unidirectional flow valve, while the outlet tube had a constricting clip attached with which the bag could be sealed. As the subject exhaled the bag inflated and breath passed through the bag to the outlet. When the subject had exhaled fully the bag therefore contained the final $200 \mathrm{ml}$ of exhaled 'alveolar' breath. A similar collection method was also used for ambient air samples except that a syringe was used to inflate the bag. 


\subsection{Alkane Analysis}

Alkane analysis was performed as previously described with minor modifications. [24-26,29] The collection bag was connected via the outlet to a $130 \mathrm{ml}$ PTFE syringe and a volume of gas removed. The syringe was then used to apply the gas sample to a thermodesorption tube (Markes Instruments, UK) containing Carbotrap 300 (Markes Instruments, UK), prior to analysis using PerkinElmer autosystem XL equipped with a Turbomass mass spectrometer (Perkin-Elmer, UK). The absorbed gases were desorbed at $320^{\circ} \mathrm{C}$ onto a cold trap held at $5^{\circ} \mathrm{C}$. Secondary desorption at $350^{\circ} \mathrm{C}$ released the volatiles into a $2 \mathrm{ml} \mathrm{min}^{-1}$ stream of helium onto a $30 \mathrm{~m} \times 0.32 \mathrm{~mm}$ PLOT GQ column. The initial GC oven temperature of $45^{\circ} \mathrm{C}$ was maintained for $10 \mathrm{~min}$ and increased at $200^{\circ} \mathrm{C}$ at a rate of $14^{\circ} \mathrm{C} \mathrm{min}^{-1}$. Eluted gases were detected by electron ionisation mass spectrometry with ethane eluting at $2.6 \mathrm{~min}$, butane at $9.6 \mathrm{~min}$ and pentane at $12.1 \mathrm{~min}$, and quantified by comparison with a standard curve constructed using a C1-C6 alkane mix (Supelco, UK).

\section{RESULTS}

Breath samples were collected from 28 patients with schizophrenia who have received no psychoactive medications for at 2 - 3 weeks prior to sampling, and from 15 healthy controls, and assayed for ethane, butane and pentane. As is observed for other trace gases in breath [38,39], and for breath alkanes in our previous study [26], ethane, butane and pentane concentrations followed an apparent $\log$ normal distribution (Lilliefors test for normality of $\log$ transformed data; $P>0.05$ for all groups). Due to this finding the log of the concentration was used in the statistical analysis of the data. Ethane and pentane concentrations were elevated in the breath of patients with schizophrenia compared to healthy controls (two-tailed, unpaired t-test; $P<0.01$ ). Butane concentrations did not differ significantly between the two groups (two-tailed, unpaired t-test; $P>0.05$ ). Gender had no statistically significant effect upon the concentrations of either gas (two-tailed, unpaired t-test; $P>0.05$ ). Age did not correlate significantly $(P>0.05)$ with concentrations of each alkane (data not shown). Butane concentrations did not correlate significantly $(P<0.05)$ with ethane or pentane concentrations in either the schizophrenia (Figure 2) or healthy control groups (Pearson correlation coefficient $=0.06$ ), but ethane concentrations were correlated with pentane concentrations in the schizophrenia group (Pearson correlation coefficient $=0.59 ; P<0.001)$ but not in the healthy control group (Pearson correlation coefficient $=0.10 ; P>0.05$ ). Ambient alkane concentrations were below 1 PPBV for each gas, close to the limits of detection of the assay (approx. 0.1 PPBV), and did not differ significantly between the schizophrenia and healthy control groups (t-test; $P<0.05$ ).

\section{DISCUSSION}

Our findings confirm previous reports $[25,26]$ of elevated breath ethane concentrations in patients with schizophrenia in a different population indicating elevated oxidative damage to omega-3 PUFA. The breath concentrations observed are similar to that found in our previous investigation of schizophrenia [25,26,29], and for healthy controls are similar to that reported by other investigators. [40] In addition, we have also observed increased breath pentane concentrations in the breath of patients with schizophrenia, suggesting that omega- 6 PUFA are similarly affected. A common mechanism underlying increased breath ethane and pentane, that of oxidative damage of PUFA, is supported by the correlation between the abundance of each alkane in the schizophrenia group (no significant correlation was observed in the healthy control group but this may be due to the rather low breath alkane concentrations $<1$ PPB being closer to the limits of detection for the assay). Indeed, reduced levels of PUFA have been reported to occur in schizophrenia (for example see reference 41), an observation which may be due to elevated oxidative damage of these fatty acids in the disorder. On the other hand, ethane concentrations are not reported to be correlated with erythrocyte omega-3 PUFA abundance in

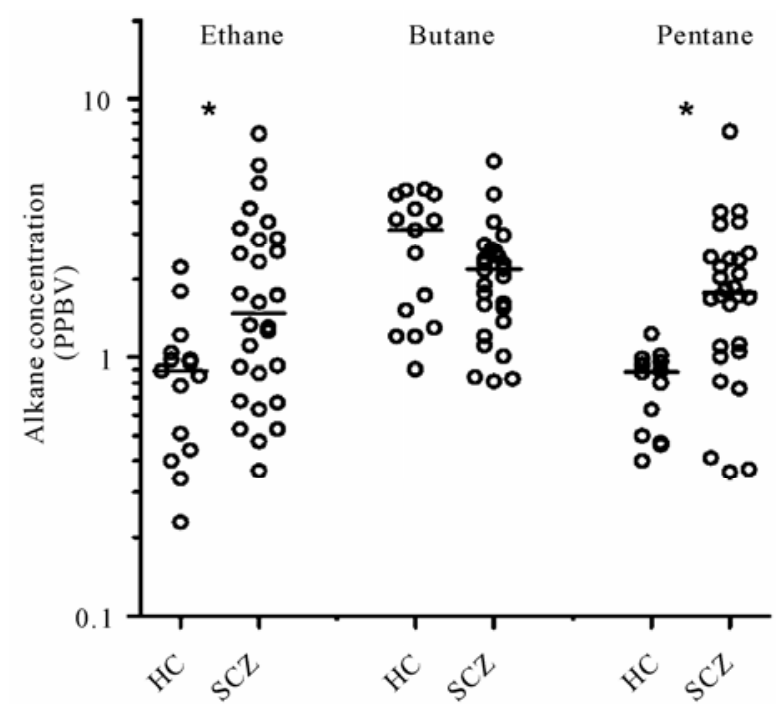

Figure 1. Breath concentrations of ethane, pentane and butane were measured in patients with schizophrenia (SCZ) and healthy controls (HC). Note the logarithmic scale of the Y-axis. The bar indicates the median value. SCZ and HC groups were compared using an unpaired t-test with *indicating $\mathrm{P}<0.01$. PPBV - parts per billion by volume. 


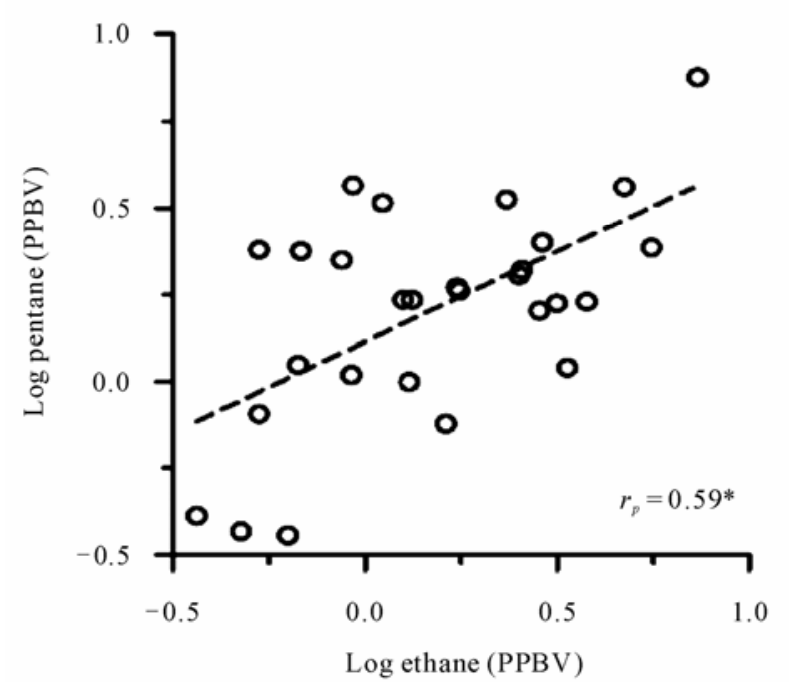

(a)

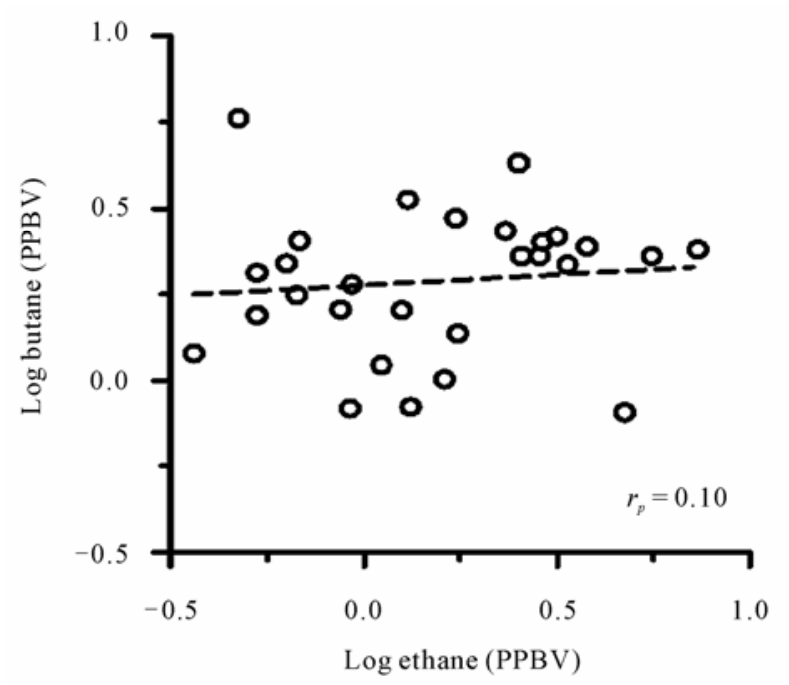

(b)

Figure 2. Correlation between alkane concentrations in breath in patients with schizophrenia. The log breath concentrations of (a) pentane or (b) butane are shown plotted against the log concentration of ethane in parts per billion by volume (PPBV). The dashed line shows the 'best fit' linear regression line along with the Pearson correlation coefficient with *indicating $\mathrm{P}<$ 0.001 .

medicated patients [26]. This suggests that other factors, such as dietary intake, may also underlie reduced fatty acid levels in addition to elevated oxidative stress [42] and that there is not a simple relationship between breath alkane concentrations and the abundance of their precursor lipids.

The magnitude of the increase in breath alkane concentrations is similar to that observed in medicated patients [26], even though our participants were unmedicated for at least 3 weeks prior to breath sampling. As such, increased breath ethane and pentane concentrations are likely not derived directly from any administered drugs, although we cannot rule out a longer term effect of any drug upon cellular metabolism given that the participants were not drug-naïve. Such findings are in agreement with data obtained using other experimental approaches which also suggest that psychoactive medication is not an important mediator of oxidative stress in patients. $[8,43]$

It is important to note that breath butane concentrations were normal in schizophrenia, and found to be uncorrelated with ethane or pentane concentrations. We had previously observed increased breath butane concentrations in schizophrenia, but reasoned that this finding was due to elevated ambient butane abundance in the sampling room used for patients, leading us to conclude that breath butane has an exogenous source. [26] However, ambient butane concentrations were very low in the current study $(<1$ PPBV) and breath butane concentrations were routinely above ambient. This does suggest a possible endogenous source for butane, as revealed by low ambient gas concentrations, although the mechanism leading to its production is presently unknown. Interestingly, in vitro chemical oxidation of erythrocytes does give rise to butane production although the chemical source is unknown [44-46]. Nevertheless, the lack of correlation with butane, as well as reports that ambient C1 - C4 alkane concentrations are correlated in polluted environmental air [47], is supportive of breath ethane and pentane are predominantly endogenous metabolic products (at least when sampled in the absence of significant alkane air pollution as in the current investigation). Notably, breath alkane concentrations were found to follow a log normal distribution, similar to that observed for other breath gases. [38,39]

Most of our subjects were smokers, the rate of which did not differ between the schizophrenia and control groups in this Indian sample. Such a finding differs from that in Western nations but is consistent with a previous report of cigarette use in this country [48]. The lack of a differential smoking rate between the two groups is an important consideration since there reports that smoking increases breath ethane levels although this is not a consistent finding $[49,50]$. We did not, however, assess the number of cigarettes smoked per day and hence cannot rule out that the schizophrenia group had a higher exposure to tobacco product use than the control group. We have previously reported, however, that there is no correlation between tobacco exposure and ethane concentrations in patients with schizophrenia [26], which supports our conclusion that increased breath alkane concentrations in schizophrenia are consequent to a metabolic change associated with the illness. 
Our data therefore offer further evidence for the occurrence of elevated oxidative stress in schizophrenia. Our findings also support the use of breath ethane and/or pentane concentration measurements as a means to monitor oxidative stress in schizophrenia. An examination of Figure 1, however, indicates that elevated ethane or pentane concentrations do not occur in all patients with schizophrenia, with many having breath concentrations close to the lower end of the control range. As such elevated ethane and pentane concentrations, and possibly oxidative stress in general, may not be a universal feature of the illness. It remains a possibility, however, that breath ethane and pentane are related to symptom severity in drug-free patients, as are other oxidative markers [43], although this was not addressed in the present study. It should be noted, however, that breath ethane concentration was not correlated with positive or negative symptoms severity in chronic, medicated patients with schizophrenia. [26] In addition to symptom severity this study also did not consider the role of diet which may differ between healthy controls and patients with schizophrenia, thereby representing a possibly confounding factor. For example, McCreadie and colleagues have reported that dietary intake of antioxidants is lower in patients with schizophrenia, a difference which could explain higher breath alkanes in the disorder. [51] Future studies could address this matter by incorporating dietary questionnaires and/or the measurement of systemic antioxidant levels.

It is presently unclear whether increased ethane and pentane concentrations and/or oxidative stress are of primary aetiological importance or are an epiphenomena. Nevertheless, a presumed increase in oxidative stress is not desirable, having been implicated in a variety of common disorders such as atherosclerosis, cancer and diabetes. [52-54] Interestingly, patients with schizophrenia have a higher risk of death from some forms of cancer, heart disease and diabetes. [55-57] One may therefore speculate that is related to increased oxidative stress in the disorder. For this reason it may be desirable to reduce oxidative stress as a precautionary measure by, for example, improving dietary antioxidant consumption, as a means to improve general health.

In summary, we have found that concentrations of ethane and pentane, but not butane, are increased in the breath of currently un-medicated patients with schizophrenia, a finding that provides further evidence in support of the disease being associated with increased oxidative stress. Such a biochemical change has been suggested to be of aetiological significance in the disorder, appearing to worsen the symptoms of the disease. Being non-invasive breath alkane analysis may be suited to monitoring of this condition, although further investigation is needed to elucidate the cause of the breath changes observed.

\section{ACKNOWLEDGEMENTS}

We thank Mr. Ivor McKenzie for technical assistance.

\section{REFERENCES}

[1] Van Os, J. and Kapur, S. (2009) Schizophrenia. Lancet, 374, 635-645. doi:10.1016/S0140-6736(09)60995-8

[2] Saha, S., Chant, D., Welham, J. and McGrath, J. (2005) A systematic review of the prevalence of schizophrenia. PLoS Medicine, 2, e141. doi:10.1371/journal.pmed.0020141

[3] Akyol, O., Herken, H., Uz, E., Fadillioğlu, E., Unal, S., Söğüt, S., Ozyurt, H. and Savaş, H.A. (2002) The indices of endogenous oxidative and antioxidative processes in plasma from schizophrenic patients. The possible role of oxidant/antioxidant imbalance. Progress in Neuropsychopharmacology and Biological Psychiatry, 26, 995-1005. doi:10.1016/S0278-5846(02)00220-8

[4] Halliwell, B. and Gutteridge, J.M. (1999) Free radicals in biology and medicine. 3rd Edition, Oxford University Press, UK.

[5] Reddy, R., Sahebarao, M.P., Mukherjee, S. and Murthy, J.N. (1991) Enzymes of the antioxidant defense system in chronic schizophrenic patients. Biological Psychiatry, 30, 409-412. doi:10.1016/0006-3223(91)90298-Z

[6] Mahadik, S.P. and Mukherjee, S. (1996) Free radical pathology and antioxidant defense in schizophrenia: A review. Schizophrenia Research, 19, 1-17.

doi:10.1016/0920-9964(95)00049-6

[7] Kuloglu, M., Ustundag, B., Atmaca, M., Canatan, B., Tezcan, A.E. and Cinkilinc, N. (2002) Lipid peroxidation and antioxidant enzyme levels in patients with schizophrenia and bipolar disorder. Cell Biochemistry and Function, 20, 171-175. doi:10.1002/cbf.940

[8] Yao, J.K., Reddy, R., McElhinny, L.G. and van Kammen, D.P. (1998) Effects of haloperidol on antioxidant defense system enzymes in schizophrenia. Journal of Psychiatry Research, 32, 385-391. doi:10.1016/S0022-3956(98)00028-4

[9] Mukerjee, S., Mahadik, S.P., Scheffer, R., Correnti, E.E. and Kelkar, H. (1996) Impaired antioxidant defense at the onset of psychosis. Schizophrenia Research, 19 19-26. doi:10.1016/0920-9964(95)00048-8

[10] Evans, D.R., Parikh, V.V., Khan, M.M., Coussons, C., Buckley, P.E. and Mahadik, S.P. (2003) Red blood cell membrane essential fatty acid metabolism in early psychotic patients following antipsychotic drug treatment. Prostaglandins Leukotrienes and Essential Fatty Acids, 69, 393-399. doi:10.1016/j.plefa.2003.08.010

[11] Herken, H., Uz, E., Ozyurt, H., Sogut, S., Virit, O. and Akyol, O. (2001) Evidence that the activities of erythrocyte free radical scavenging enzymes and the products of lipid peroxidation are increased in different forms of schizophrenia. Molecular Psychiatry, 6, 66-73. doi:10.1038/sj.mp.4000789

[12] Yao, J.K., Reddy, R.D. and van Kammen, D.P. (1999) 
Human plasma glutathione peroxidase and symptom severity in schizophrenia. Biological Psychiatry, 45, 1512-1515. doi:10.1016/S0006-3223(98)00184-X

[13] Ranjekar, P.K., Hinge, A., Hegde, M.V., Ghate, M., Kale, A., Sitasawad, S., Wagh, U.V., Debsikdar, V.B. and Mahadik, S.P. (2003) Decreased antioxidant enzymes and membrane essential polyunsaturated fatty acids in schizophrenic and bipolar mood disorder patients. Psychiatry Research, 121, 109-122. doi:10.1016/S0165-1781(03)00220-8

[14] Ustundag, B., Atmaca, M., Kirtas, O., Selek, S., Metin, K. and Tezcan, E. (2006) Total antioxidant response in patients with schizophrenia. Psychiatry and Clinical Neuroscience, $\mathbf{6 0}, 458-464$. doi:10.1111/j.1440-1819.2006.01532.x

[15] Yao, J.K., Reddy, R., McElhinny, L.G. and van Kammen, D.P. (1998) Reduced status of plasma total antioxidant capacity in schizophrenia. Schizophrenia Research, 32, 1-8. doi:10.1016/S0920-9964(98)00030-9

[16] Virit, O., Altindag, A., Yumru, M., Dalkilic, A., Savas, H.A., Selek, S., Erel, O. and Herken, H. (2009) A defect in the antioxidant defense system in schizophrenia. Neuropsychobiology, 60, 87-93. doi:10.1159/000239684

[17] Nishioka, N. and Arnold, S.E. Evidence for oxidative DNA damage in the hippocampus of elderly patients with chronic schizophrenia. American Journal of Geriatric Psychiatry, 12, 167-175.

[18] Young, J., McKinney, S.B., Ross, B.M., Wahle, K.W. and Boyle, S.P. (2007) Biomarkers of oxidative stress in schizophrenic and control subjects. Prostaglandins Leukotrienes and Essential Fatty Acids, 76, 73-85.

[19] Dietrich-Muszalska, A., Olas, B., Głowacki, R. and Bald, E. (2009) Oxidative/nitrative modifications of plasma proteins and thiols from patients with schizophrenia. Neuropsychobiology, 59, 1-7. doi:10.1159/000202822

[20] Dietrich-Muszalska, A. and Olas, B. (2007) Isoprostenes as indicators of oxidative stress in schizophrenia. World Journal of Biological Psychiatry, 11, 1-7.

[21] Wang, J.F., Shao, L., Sun, X. and Young, L.T. (2009) Increased oxidative stress in the anterior cingulate cortex of subjects with bipolar disorder and schizophrenia. Bipolar Disorders, 11, 523-529. doi:10.1111/j.1399-5618.2009.00717.x

[22] Steullet, P., Cabungcal, J.H., Kulak, A., Kraftsik, R., Chen, Y., Dalton, T.P., Cuénod, M. and Do, K.Q. (2010) Redox dysregulation affects the ventral but not dorsal hippocampus: Impairment of parvalbumin neurons, gamma oscillations, and related behaviors. Journal of Neuroscience, 30, 2547-2558.

doi:10.1523/JNEUROSCI.3857-09.2010

[23] Cabungcal, J.H., Nicolas, D., Kraftsik, R., Cuénod, M., Do, K.Q. and Hornung, J.P. (2006) Glutathione deficit during development induces anomalies in the rat anterior cingulate GABAergic neurons. Neurobiology of Disease, 22, 624-637.

[24] Puri, B.K., Counsell, S.J., Ross, B.M., Hamilton, G., Bustos, M.G. and Treasaden, I.H. (2008) Evidence from in vivo 31-phosphorus magnetic resonance spectroscopy phosphodiesters that exhaled ethane is a biomarker of cerebral n-3 polyunsaturated fatty acid peroxidation in humans. BMC Psychiatry, 8.

doi:10.1016/j.nbd.2006.01.003
[25] Puri, B.K., Ross, B.M. and Treasaden, I.H. (2008) Increased levels of ethane, a non-invasive, quantitative, direct marker of n-3 lipid peroxidation, in the breath of patients with schizophrenia. Progress in Neuropsychopharmacology and Biological Psychiatry, 32, 858-862. doi:10.1016/j.pnpbp.2008.01.001

[26] Ross, B.M., Maxwell, R. and Glen, I. (2011) Increased breath ethane levels in medicated patients with schizophrenia and bipolar disorder are unrelated to erythrocyte omega-3 fatty acid abundance. Progress in Neuropsychopharmacology and Biological Psychiatry, 35, 436-453.

[27] Dillard, C.J., Dumelin, E.E. and Tappel, A.L. (1977) Effect of dietary vitamin $\mathrm{E}$ on expiration of pentane and ethane by the rat. Lipids, 12, 109-114. doi:10.1007/BF02532981

[28] Wade, C.R. and van Rij, A.M. (1985) In vivo lipid peroxidation in man as measured by the respiratory excretion of ethane, pentane, and other low-molecular-weight hydrocarbons. Analytical Biochemistry, 150, 1-7. doi:10.1016/0003-2697(85)90433-6

[29] Ross, B.M., McKenzie, I., Glen, I. and Bennett, C.P. (2003) Increased levels of ethane, a non-invasive marker of n-3 fatty acid oxidation, in breath of children with attention deficit hyperactivity disorder. Nutritional Neuroscience, 6, 277-281. doi:10.1080/10284150310001612203

[30] Amann, A., Spaněl, P. and Smith, D. (2007) Breath analysis: The approach towards clinical applications. Mini Reviews in Medicinal Chemistry, 7, 115-129. doi:10.2174/138955707779802606

[31] Bloor, R.N., Spanĕl, P. and Smith, D. (2006) Quantification of breath carbon disulphide and acetone following a single dose of disulfiram (antabuse) using selected ion flow tube mass spectrometry (SIFT-MS). Addiction Biology, 11, 163-169.

doi:10.1111/j.1369-1600.2006.00015.x

[32] Giacomuzzi, S.M., Riemer, Y., Pavlic, M., Schmid, A., Hinterhuber, H. and Amann, A. (2009) Applications of breath gas analysis in addiction medicine-preliminary results. Substance Use and Misuse, 44, 301-304. doi:10.1080/10826080802344864

[33] Miljevic, C., Nikolic, M., Nikolic-Kokic, A., Jones, D.R., Niketic, V., Lecic-Tosevski, D. and Spasic, M.B. (2010) Lipid status, anti-oxidant enzyme defence and haemoglobin content in the blood of long-term clozapine-treated schizophrenic patients. Progress in Neuropsychopharmacology and Biological Psychiatry, 34, 303-307. doi:10.1016/j.pnpbp.2009.11.024

[34] Singh, O.P., Chakraborty, I., Dasgupta, A. and Datta, S. (2008) A comparative study of oxidative stress and interrelationship of important antioxidants in haloperidol and olanzapine treated patients suffering from schizophrenia. Indian Journal Psychiatry, 50, 171-176. doi:10.4103/0019-5545.43627

[35] Walss-Bass, C., Weintraub, S.T., Hatch, J., Mintz, J. and Chaudhuri, A.R. (2008) Clozapine causes oxidation of proteins involved in energy metabolism: A possible mechanism for antipsychotic-induced metabolic alterations. International Journal of Neuropsychopharmacology, 11, 1097-1104. doi:10.1017/S1461145708008882

[36] Martins, M.R., Petronilho, F.C., Gomes, K.M., Dal-Pizzol, F., Streck, E.L. and Quevedo, J. (2008) Antipsycho- 
tic-induced oxidative stress in rat brain. Neurotoxicolgy Research, 13, 63-69. doi:10.1007/BF03033368

[37] American Psychiatric Association. (1994) Diagnostic and statistical manual of mental health disorders. 4th Edition, American Psychiatric Publishing, Washington, DC.

[38] Smith, D., Španěl, P., Enderby, B., Lenney, W., Turner, C. and Davies, S.J. (2010) Isoprene levels in the exhaled breath of 200 healthy pupils within the age range 7-18 years studied using SIFT-MS. Journal of Breath Research, 4, 017101.

[39] Smith, D., Turner, C. and Spaněl, P. (2007) Volatile metabolites in the exhaled breath of healthy volunteers: Their levels and distributions. Journal of Breath Research, 1, 014004 . 2007doi:10.1088/1752-7155/1/1/014004

[40] Abela, J.E., Skeldon, K.D., Stuart, R.C. and Padgett, M.J. (2009) Exhaled ethane concentration in patients with cancer of the upper gastrointestinal tract - A proof of concept study. Bioscience Trends, 3, 110-114.

[41] Reddy, R.D., Keshavan, M.S. and Yao, J.K. (2004) Reduced red blood cell membrane essential polyunsaturated fatty acids in first episode schizophrenia at neuroleptic-naive baseline. Schizophrenia Bulletin, 30, 901-911.

[42] Peet, M., Shah, S., Selvam, K. and Ramchand, C.N. (2004) Polyunsaturated fatty acid levels in red cell membranes of unmedicated schizophrenic patients. World Journal of Biological Psychiatry, 5, 92-99. doi:10.1080/15622970410029917

[43] Raffa, M., Mechri, A., Othman, L.B., Fendri, C., Gaha, L. and Kerkeni, A. (2009) Decreased glutathione levels and antioxidant enzyme activities in untreated and treated schizophrenic patients. Progress in Neuropsychopharmacology and Biological Psychiatry, 33, 1178-1183. doi:10.1016/j.pnpbp.2009.06.018

[44] Clemens, M.R., Remmer, H. and Waller, H.D. (1984) Phenylhydrazine-induced lipid peroxidation of red blood cells in vitro and in vivo: Monitoring by the production of volatile hydrocarbons. Biochemical Pharmacology, 33, 1715-1718. doi:10.1016/0006-2952(84)90338-1

[45] Clemens, M.R. and Remmer, H. (1982) Volatile alkanes produced by erythrocytes: An assay for in vitro studies on lipid peroxidation. Blut, 45, 329-335. doi:10.1007/BF00319527

[46] Einsele, H., Clemens, M.R. and Remmer, H. (1987) In vitro aging of red blood cells and lipid peroxidation. Archives of Toxicology, 60, 163-166.
doi:10.1007/BF00296972

[47] Fowler, D., Coyle, M. and Anderson, R. (1997) Ozone in the United Kingdom. The Fourth Report of the Oxidants Review Group. Department of the Environment, Transport and the Regions, London, UK.

[48] Srinivasan, T.N. and Thara, R. (2002) Smoking in schizophrenia - All is not biological. Schizophrenia Research, 56, 67-74. doi:10.1016/S0920-9964(01)00187-6

[49] Puri, B.K., Treasaden, I.H., Cocchi, M., Tsaluchidu, S., Tonello, L. and Ross, B.M. (2008) A comparison of oxidative stress in smokers and non-smokers: An in vivo human quantitative study of n-3 lipid peroxidation. $M C$ Psychiatry, 8, Suppl 1:S4. doi:10.1186/1471-244X-8-S1-S4

[50] Habib, M.P., Clements, N.C. and Garewal, H.S. (1995) Cigarette smoking and ethane exhalation in humans. American Journal of Respiratory and Critical Care Medicine, 151, 1368-1372.

[51] McCreadie, R.G. (2003) Diet, smoking and cardiovascular risk in people with schizophrenia. British Journal of Psychiatry, 183, 534-539. doi:10.1192/bjp.183.6.534

[52] Wei, W., Liu, Q., Tan, Y., Liu, L., Li, X. and Cai, L. Oxidative stress, diabetes, and diabetic complications. Hemoglobin, 33, 370-377. doi:10.3109/03630260903212175

[53] Bruckdorfer, K.R. (2008) Antioxidants and CVD. Proceedings of the Nutrition Society, 67, 214-222. doi:10.1017/S0029665108007052

[54] Visconti, R. and Grieco, D. (2009) New insights on oxidative stress in cancer. Current Opinions in Drug Discovery Development, 12, 240-245.

[55] Bushe, C.J., Bradley, A.J., Wildgust, H.J. and Hodgson, R.E. (2009) Schizophrenia and breast cancer incidence: A systematic review of clinical studies. Schizophrenia Research, 114, 6-16. doi:10.1016/j.schres.2009.07.012

[56] Koponen, H., Alaräisänen, A., Saari, K., Pelkonen, O., Huikuri, H., Raatikainen, M.J., Savolainen, M. and Isohanni, M. (2008) Schizophrenia and sudden cardiac death: A review. Nordic Journal of Psychiatry, 62, 342-345. doi:10.1080/08039480801959323

[57] Suvisaari, J., Perälä, J., Saarni, S.I., Härkänen, T., Pirkola, T., Joukamaa, M., Koskinen, S., Lönnqvist, J. and Reunanen, A. (2008) Type 2 diabetes among persons with schizophrenia and other psychotic disorders in a general population survey. European Archives of Psychiatry and Clinical Neuroscience, 258, 129-136. 